\title{
Estudo comparativo entre larvitrampas e ovitrampas para avaliação da presença de Aedes aegypti (Diptera: Culicidae) em Campo Grande, Estado do Rio de Janeiro
}

\author{
Comparative study between larvitraps and ovitraps for evaluating the presence of \\ Aedes aegypti (Diptera: Culicidae) in Campo Grande, State of Rio de Janeiro
}

\author{
Vanderlei Campos Silva ${ }^{1}$, Nicolau Maués Serra-Freire ${ }^{2}$, Júlia dos Santos Silva ${ }^{3,4}$, Paulo \\ Oldemar Scherer ${ }^{5}$, Iram Rodrigues ${ }^{5}$, Sergio Pereira Cunha ${ }^{5}$ e Jeronimo Alencar $^{3}$
}

\begin{abstract}
RESUMO
Objetivando-se avaliar a eficiência de armadilhas no monitoramento de vetores de dengue e febre amarela no Rio de Janeiro, foram utilizadas simultaneamente, 12 larvitrampas e 12 ovitrampas ao longo de 13 semanas. Resultados mostraram que as larvitrampas apresentam maior capacidade de positivar, destacando-se como importante ferramenta no monitoramento de vigilância vetorial.
\end{abstract}

Palavras-chaves: Controle de mosquitos. Ecologia de vetores. Aedes. Dengue.

\begin{abstract}
With the objective of evaluating the efficiency of traps for monitoring dengue and yellow fever vectors in Rio de Janeiro, 12 larvitraps and 12 ovitraps were used simultaneously for 13 weeks. The results indicated that the larvitraps presented greater capacity for positive findings, thereby highlighting it as an important monitoring tool for vector surveillance.
\end{abstract}

Key-words: Mosquito Control. Vector ecology. Aedes. Dengue.

Nas Américas, a dengue constitui uma doença de aspecto principalmente urbano, no qual os agentes etiológicos fazem parte do ciclo que envolve o mosquito Aedes aegypti (Linnaeus) e o ser humano ${ }^{7}$. 0 Aedes aegypti é considerado o principal vetor de dengue e febre amarela do mundo e apresenta alta eficiência na vetoração em populações humanas, fato que é atribuído em parte aos seus hábitos sinantrópicos ${ }^{12}$. Essa espécie está estreitamente relacionada ao homem, utilizando-se dos recipientes descartados por este, como criadouros para suas formas imaturas. Seu comportamento mostra preferência por recipientes de cor escura, contendo água limpa, pobre em matéria orgânica e disposto a sombra no intra ou peridomicílio ${ }^{1}$. A comparação entre a pesquisa larvária e armadilha de oviposição para detecção de Aedes aegypti vem sendo utilizada em vários programas de controle em diversos países. No Brasil, a Secretaria de Vigilância em Saúde ainda não adotou este método na sua rotina. Entretanto, vários autores têm

\footnotetext{
1. Laboratório de Transmissores de Leishmanioses, Fundação Oswaldo Cruz, Rio de Janeiro, RJ. 2. Laboratório de Ixodides, Fundação Oswaldo Cruz, Rio de Janeiro, RJ. 3. Laboratório de Diptera, Fundação Oswaldo Cruz, Rio de Janeiro, RJ. 4. Programa de Pós-Graduação em Zoologia, Museu Nacional, Universidade Federal do Rio de Janeiro, Rio de Janeiro, RJ. 5. Fundação Nacional de Saúde, Rio de Janeiro, RJ.

Endereço para correspondência: Dr. Jeronimo Alencar. Laboratório de Diptera/ FIOCRUZ. Av. Brasil 4365, Manguinhos, 21040-360 Rio de Janeiro, RJ.

Tel: $55212562-1450$.

e-mail: jalencar@ioc.fiocruz.br

Recebido para publicação em 17/08/2009

Aceito em 19/10/2009
}

demonstrado que essa técnica é sensivel ao monitoramento desses vetores quando a pesquisa larvária nas unidades domiciliares não os detecta ${ }^{12}{ }^{10}$. A utilização da armadilha de ovitrampa como ferramenta para detectar a presença de população de Aedes aegypti foi primeiramente proposta por Fay \& Perry ${ }^{6}$. Fay \& Eliason ${ }^{5}$, em 1966, compararam essas duas armadilhas, e verificaram que as ovitrampas foram superiores em relação às outras. Reiter cols ${ }^{11}$, em 1991, verificaram que adicionando feno à ovitrampa, estas tiveram seus índices aumentados se comparados com aquelas sem tal substância.

O presente estudo teve como objetivo avaliar comparativamente estas armadilhas no monitoramento de Aedes aegypti em bairro situado na zona oeste do município do Rio de Janeiro, Campo Grande, durante treze semanas. 0 bairro de Campo Grande tem coordenadas de $22^{\circ} 53^{\prime} \mathrm{S}$ e $43^{\circ} 33^{\prime} \mathrm{W}$, dista cerca de $50 \mathrm{~km}$ do centro do município do Rio de Janeiro, tem área territorial de $171,67 \mathrm{~km}^{2}$, ocupada por cerca de 297.494 habitantes (IBGE, 2000). A escolha pelo bairro de Campo Grande foi levada em conta devido aos elevados índices de incidência de dengue e alta infestação de Aedes aegypti.

As armadilhas utilizadas nesse experimento foram: ovitrampas, constituídas de um recipiente artificial de plástico, de formato arredondado e cor preta, medindo $9 \mathrm{~cm}$ de altura por $11 \mathrm{~cm}$ de diâmetro, onde nessas armadilhas as paletas utilizadas eram de compensado de eucatex com $5 \mathrm{~mm}$ de espessura, $12,5 \mathrm{~cm}$ de comprimento por $2 \mathrm{~cm}$ de largura. Cada paleta foi utilizada apenas 
uma vez, a fim de evitar erro na contagem dos ovos e fixada nas ovitrampas com auxílio de um clipe, posicionada verticalmente neste recipiente contendo aproximadamente $300 \mathrm{ml}$ de água destilada, onde foi adicionado serrapilheira; e larvitrampas, por sua vez consistiam de pneus cortados ao meio e penduradas a 1,60m de altura do solo em local sombreado e afastado das ovitrampas por cerca de $6 \mathrm{~m}$. A cada inspeção das armadilhas, as paletas eram identificadas e acondicionadas em sacos plásticos individuais e levadas ao laboratório para identificação e contagem dos ovos, e ainda, quando necessário, completava-se o volume de água. As larvas das larvitrampas eram acondicionadas em tubos, contendo álcool a 70\% e levadas ao laboratório para identificação e contagem. Após cada busca ativa, as larvitrampas eram lavadas e escovadas em água corrente e flambadas na intenção de evitar erro no experimento. Em seguida adicionava-se água até atingir o nível inicial.

As pesquisas foram realizadas no horário da manhã, com intervalos de dois dias, de 27 de agosto a 26 de novembro de 2005 , tendo como base para as observações do horário de oviposição, os referidos por Chadee \& Corbert ${ }^{2}$, em estudos desenvolvidos com Aedes albopictus (Skuse). As armadilhas foram localizadas concomitantemente em imóveis residenciais de acordo com Jakob \& Bevier$^{8}$. Em virtude da grande dificuldade para identificar as espécies através do ovo, estas identificações foram realizadas preferencialmente em larvas de $3^{\circ}$ ou $4^{\circ}$ instar, através de um microscópio (Zeiss®), após eclosão espontânea em laboratório.

Para avaliar a eficiência das armadilhas das diferentes metodologias, foram calculados os coeficientes de dominância, abundância e intensidade média de captura. As larvitrampas apresentaram maior positividade. A comparação entre indicadores entomológicos, para as seis armadilhas de cada tipo, mostrou diferenças não significativas $(\mathrm{p}<0,05)$ entre as armadilhas, considerando-se então como válida a constatação de que nas áreas trabalhadas o Aedes aegypti é a espécie dominante, com abundância de aproximadamente 17 larvas e quatro ovos por semana de estudo, e cerca de 220 larvas, mais 53 ovos de positividade, em cada local de criação no período investigado. Resultado também constatado para Aedes albopictus, onde foi observado desempenho similar utilizando esse tipo de armadilhas $\mathrm{f}_{\text {tab. } 5 \%}=1,91>\mathrm{f}_{\text {calc }}$, e cerca de três larvas/semana.

Dentre as semanas pesquisadas, utilizando as armadilhas de larvitrampas, a décima terceira apresentou a maior abundância de espécimes encontrados, com 29,4\%, enquanto a oitava semana foi a menor com 2,5\% do total. As semanas de experimento três e quatro foram as que apresentaram maior número de larvitrampas positivas, totalizando dez. Já a semana nove, foi encontrada apenas uma armadilha positiva. Para as armadilhas ovitrampas, foi constatado o melhor desempenho de positividade apenas na décima semana de experimento, perfazendo 14,3\% dos ovos encontrados nas 13 semanas de estudos. Nas semanas dois e treze, concentraram-se as armadilhas com maior positividade (quatro em cada uma), e menor número de espécimes aconteceu na 11 semana, correspondendo a $0,95 \%$ do total.

Os casos de dengue durante o período da pesquisa entomológica tiveram as ocorrências concentradas no mês de outubro, resultado que coincide com a maior positividade de
Aedes aegypti na área estudada. É importante destacar que este período sazonal para o município do Rio de Janeiro, corresponde às estações de inverno e primavera, período de baixos índices pluviométricos.

Neste estudo, foi constatado que as armadilhas de larvitrampas tiveram maior capacidade de positivar, quando comparadas com as armadilhas de ovitrampas em quase todo período do monitoramento entomológico. Observações realizadas por Cheng cols ${ }^{9}$, em 1982, e Lima cols ${ }^{9}$, em 1989, comparando diferentes tipos de armadilhas, relataram resultados semelhantes com as larvitrampas mais atrativas. Lima cols ${ }^{9}$ consideram que a superfície disponível e o volume de água influenciam a oviposição, fato que explica a maior eficiência da armadilha de pneu. Chadee ${ }^{2}$, em 1990, constatou que as armadilhas de ovitrampas apresentam-se como boa ferramenta para detecção precoce de vetores de dengue e febre amarela, fato que não foi comprovado no presente estudo. Entretanto, Marques cols $^{10}$, em 1993, relataram que armadilhas ovitrampas positivaram-se mesmo na presença de criadouros naturais e possuíram eficiência superior as larvitrampas. No estudo, estes autores utilizaram nas armadilhas: paletas de eucatex de $20 \mathrm{~cm} \times 05 \mathrm{~cm}$ e vasos plásticos pretos de $15 \mathrm{~cm}$ de diâmetro por $15 \mathrm{~cm}$ de profundidade. É possível que a diferença na confecção das armadilhas tenha influenciado a diferença de resultados, já que as paletas de eucatex apresentavam uma área menor de exposição, formada de $5 \mathrm{~mm}$ de espessura e $12,5 \mathrm{~cm}$ de comprimento por $2 \mathrm{~cm}$ de largura.

\section{REFERÊNCIAS}

1. Braga IA, Gomes AC, Nelson M, Mello RCG, Bergamachi DP, Souza JMP. Comparação entre pesquisa larvária e armadilha de oviposição, para detecção de Aedes aegypti. Revista da Sociedade Brasileira de Medicina Tropical 33: 347-353, 2000.

2. Chadee DD. Métodos de evaluación de la población de Aedes aegypti y tratamientos con insecticidas en uma población de Trinidad, Antillas. Boletín de la Oficina Sanitaria Panamericana 109: 350-358, 1990.

3. Cheng M, Ho B, Bartnett RE, Goodwin N. Role of a modified ovitrap in the control of Aedes aegypti in Houston, Texas, USA. Bulletin of the World Health Organization 60: 291-296, 1982.

4. Connor ME, Monroe WM. Stegomyia indices and their value in yellow fever control. The American Journal of Tropical Medicine and Hygiene 3: 9-19, 1923.

5. Fay RW, Eliason DA. A preferred oviposition sites as a surveillance method for Aedes aegypti. Mosquito News 26: 531-535, 1966.

6. Fay RW, Perry AS. Laboratory studies of ovipositional preferences of Aedes aegypti. Mosquito News 25: 276-281, 1965.

7. Forattini OP. Culicidologia Médica: Identificação, Biologia, Epidemiologia. Volume II. Editora da Universidade de São Paulo, São Paulo, 2002.

8. Jakob WL, Bevier GA. Application of ovitraps in the U.S. Aedes aegypti eradication program. Mosquito News 29: 55-62, 1969.

9. Lima MM, Amaral RS, Aragão MB. Estudo comparativo da eficácia de armadilhas para Aedes aegypti. Cadernos de Saúde Pública 5: 143-150, 1989.

10. Marques CCA, Marques GRAM, Brito M, Santos-Neto LG, Ishibashi VC, Gomes FA. Estudo comparativo de eficácia de larvitrampas e ovitrampas para vigilância de vetores de dengue e febre amarela. Revista de Saúde Pública 27: 237-241, 1993.

11. Reiter P, Amador MA, Colon N. Enhancement of the CDC ovitrap with hay infusions for daily monitoring of Aedes aegypti populations. Journal of the American Mosquito Control Association 7: 52-55, 1991.

12. Scott TW, Chow E, Strickman D, Kittaypong P, Wirtz RA, Lorenz LH, Edman JD. Blood-feeding patterns of Aedes aegypti (Diptera: Culicidae) collected in a rural Thai village. Journal of Medical Entomology 30: 922-927, 1993. 